\title{
TEMPORAL CORRELATION OF METADATA ERRORS FOR COMMERCIAL SATELLITE IMAGES: REPRESENTATION AND EFFECTS ON STEREO EXTRACTION ACCURACY
}

\author{
J.T. Dolloff, H.J. Theiss \\ InnoVision Basic and Applied Research Office, Sensor Geopositioning Center, National Geospatial-Intelligence Agency (contractor) \\ PA Case 12-138, 7500 GEOINT Dr, Springfield, VA 22150 - (john.t.dolloff, henry.j.theiss).ctr@nga.mil
}

Commission I, WG I/4

KEYWORDS: satellite, image, metadata, accuracy, correlation, extraction, estimation, triangulation

\begin{abstract}
:
Extraction of ground points using a basic stereo pair of commercial satellite electro-optical images typically yields vertical errors much smaller than expected. In particular, the magnitude of vertical errors relative to horizontal errors is significantly smaller than expected based on imaging geometry (convergence angle, etc.) alone. This paper suggests that temporal correlation or similarity of metadata (sensor position, attitude) errors between two same-pass images is the major cause of this phenomenon. It discusses the sources of temporal correlation, how it can be represented, and how an optimal ground point extraction algorithm detailed in the paper uses this representation in order to provide the best possible 3D location and corresponding $3 \times 3$ error covariance for reliable predicted solution accuracy. This paper also provides an estimate for temporal correlation, approximately 0.70 (70\%), and explains how this value was derived based on the ratio of measured $0.9 \mathrm{p}$ vertical errors to measured $0.9 \mathrm{p}$ horizontal errors compiled over many stereo pairs and ground truth points as described in various papers in the literature. As demonstrated in this paper, based on simulation and error propagation for typical stereo geometry, if this correlation is not accounted for, predicted $0.9 \mathrm{p}$ vertical error is approximately $60 \%$ too large. Knowledge of temporal correlation is essential for reliable stereo accuracy prediction as well as proper modeling of a priori metadata uncertainty in the support of metadata adjustment in a value-added process, such as registration to sparse control or a block adjustment.
\end{abstract}

\section{INTRODUCTION}

\subsection{Sensor Support Data and Corresponding Errors}

The sensor support data (image metadata) for commercial satellite electro-optical imagery consists primarily of a time series of sensor position and attitude. This data is required for the extraction of a ground point identified and measured in an image (mono extraction) or in a stereo pair of images (stereo extraction). This data contains errors, which can be thought of as a stochastic process that is correlated in time, typically the closer in time, the higher the correlation or similarity between two elements in the time series, e.g., between sensor x-position error at time $\mathrm{t} 1$ and sensor $\mathrm{x}$-position error at time $\mathrm{t} 1+90$ seconds. Thus, the support data for a stereo pair of images taken on the same-pass is temporally correlated, which affects the accuracy of stereo extraction, just as imaging geometry (convergence angle, etc.) affects accuracy, as will be demonstrated later.

The above description is relative to a physical sensor model and its support data; support data for a corresponding rational polynomial coefficients (RPC) sensor model consists primarily of a ground-to-image polynomial. However, this polynomial is generated from the physical sensor model; thus, the effect of RPC errors on extraction is the same as that for the physical sensor model, plus the addition of polynomial fit error. Remaining detailed analysis will be relative to the physical sensor model, unless specifically stated otherwise. However, overall conclusions are applicable to both sensor models, since one is derived from the other.

\subsection{Optimal WLS Stereo Extraction}

An optimal Weighted-Least Squares (WLS) stereo extraction algorithm solves for both a 3D ground location and a $3 \times 3$ error covariance matrix that predicts solution accuracy, i.e., the expected magnitude of solution error and derived metrics. If it does not take the temporal correlation of sensor support data errors into account, accuracy predictions are incorrect. Similarly, if value-added processes are performed to correct the sensor support data, such as registration of the stereo pair to a sparse set of ground control, or as part of a block adjustment (aka triangulation), these processes can only be optimal if $a$ priori sensor support data uncertainty is specified, including the temporal correlation of underlying errors.

\subsection{Related References}

Applicable references regarding basic (uncontrolled) commercial satellite stereo accuracy include image vendors (DigitalGlobe, 2011) and (GeoEye, 2011), and recent accuracy assessments for WorldView-1 and GeoEye-1 involving numerous stereo pair samples, such as (Breshnahan, 2010a), (Breshnahan, 2010b), (Dolloff, 2010), and (Mulawa, 2010). In general, temporal correlation of sensor support data is not discussed in these references; although (Dolloff, 2010) discusses it briefly. In addition, (Dolloff, 2004) discusses the need and methods to account for temporal correlation in optimal geopositioning algorithms, including WLS stereo extraction, but does not present quantitative results based on real-world data. 


\subsection{Temporal Correlation}

This paper presents an optimal WLS stereo extraction algorithm that accounts for temporal correlation, given an estimate for its value. In addition, analysis is presented that estimates the appropriate value of temporal correlation for commercial satellite same-pass imagery using real data.

As presented via simulation results, and for a given imaging geometry, the degree of temporal correlation affects the ratio of solution predicted $0.9 \mathrm{p}$ vertical error (LE) to predicted $0.9 \mathrm{p}$ horizontal error (CE). Both LE and CE are computed from the solution's error covariance matrix and assume a mean zero, Gaussian (normal) joint distribution of errors. And comparing these ratios to the ratios of measured errors, an estimate of temporal correlation is obtained. A comparison is then made between the values of $\mathrm{LE}$ and $\mathrm{CE}$ computed properly accounting for temporal correlation and those mistakenly computed assuming uncorrelated sensor support data errors. Sensitivities are also included, as well as a discussion on how to best represent temporal correlation as a function of time between images.

\subsection{Paper Roadmap}

Section 2 of this paper presents a simulated example of a simple scalar stochastic process and corresponding temporal correlation as background material. Recall that sensor support data errors are represented as stochastic processes. Section 3 then presents the optimal WLS stereo extraction algorithm, which accounts for the effects of imaging geometry, mensuration error, sensor support data error, and the temporal correlation of sensor support data error between images. Quantitative statistical descriptions of the various error sources used in subsequent analyses are included. Section 4 then details the more familiar effects of imaging geometry (only) on predicted solution accuracy. Section 5 details the effects of the various error sources; in particular, predicted accuracy is presented as a function of assumed temporal correlation value. An estimated value of $70 \%$ is then derived for temporal correlation based on real data. Section 6 discusses various sources of temporal correlation and its functional representation. Section 7 presents conclusions.

\section{STOCHASTIC PROCESS EXAMPLE}

The following equations describe a scalar, first order, discrete Gauss-Markov stochastic process as background material. It is a mean-zero, wide-sense stationary stochastic process described as follows, where $E\{\}$ is the expectation operator (Gelb, 1974):

$$
\begin{aligned}
& x_{n+1}=\left(e^{-d t / T}\right) x_{n}+w_{n} \\
& E\left\{x_{n}^{2}\right\} \equiv \sigma_{x}^{2}>0 \\
& E\left\{w_{n}^{2}\right\}=\sigma_{x}^{2}\left(1-e^{-\frac{2 d t}{T}}\right) \\
& \rho_{x}(m d t)=e^{-m d t / T}
\end{aligned}
$$

where

$x_{n}$ is the stochastic process at time $t_{n}$ (meters);

$T>0$ the corresponding time constant (seconds); $d t=\left|t_{n}-t_{n-1}\right|$ (seconds)

$w_{n}$ the mean-zero Gaussian white noise driving process;

$\sigma_{x}$ the stochastic process's steady state one-sigma value;

$\rho_{x}(m d t)=E\left\{x_{n} x_{n+m}\right\} / \sigma_{x}^{2}$ is the temporal correlation function.

In general, the temporal correlation function is a normalized function with unit-less values greater than -1 and less than or equal to +1 . For the Gauss-Markov process in particular, its values are greater than 0 and less than or equal to +1 .

Figure 1 presents a simulated example of the stochastic process ( $\sigma_{x}=1, T=240$ seconds) and Figure 2 its corresponding $a$ priori temporal correlation function $\rho_{x}(m d t)$. Two samples close in time (e.g. 90 seconds) have high similarity or high positive temporal correlation.

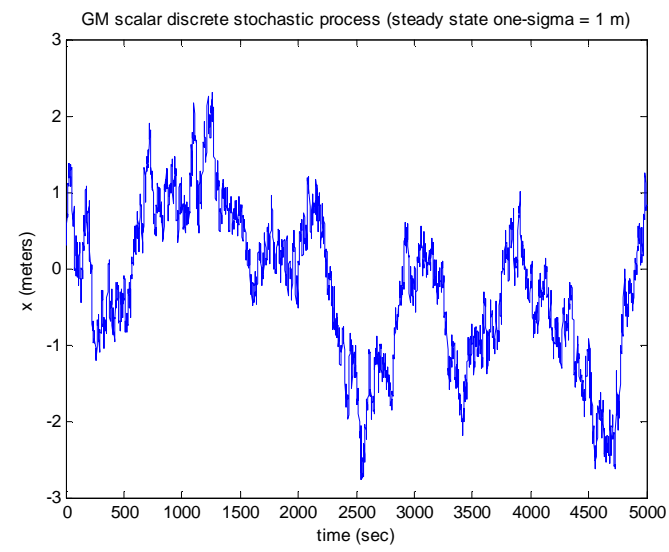

Figure 1. Stochastic process realization

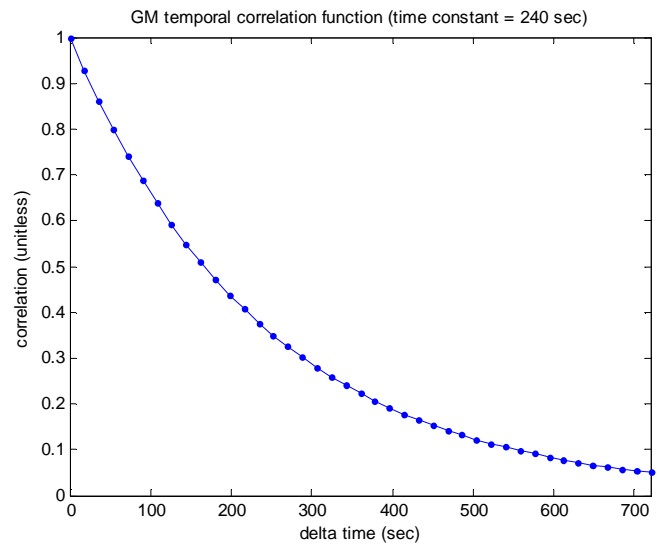

Figure 2. Stochastic process temporal correlation function

\section{WLS STEREO SOLUTION ALGORITHM}

\subsection{General Solution Equations}

The following presents the optimal WLS stereo extraction algorithm. Although corrections to sensor support data are not included by definition, its uncertainty, including temporal 
correlation, is accounted for in the measurement weight matrix $W$.

$\hat{X}=X_{0}+P_{X} B_{X}^{T} W\left(M-M_{0}\right)$

$P_{X}=\left(B_{X}^{T} W B_{X}\right)^{-1}$

$W=\left(B_{S} C_{S} B_{S}^{T}+\Sigma\right)^{-1}$

where

$\hat{X}$ is the $3 x l$ solution or best estimate of 3 D location with units of meters;

$P_{X} \equiv E\left\{\epsilon \hat{X} \in \hat{X}^{T}\right\}$ is the corresponding solution $3 \times 3$ error covariance matrix, the basis for predicted accuracy, with units of meters-squared, and where the expected value of solution error $E\{\epsilon \hat{X}\}=0$;

$W$ is the $4 x 4$ measurement weight matrix, the inverse of the sum of the mensuration error covariance and the support data error covariance projected to image space;

Supporting definitions are:

$X=\left[\begin{array}{lll}x & y & z\end{array}\right]^{T}$ is the $3 x 1$ ground location for solution in a local tangent plane coordinate system;

$M=\left[\begin{array}{llll}l 1 & s 1 & l 2 & s 2\end{array}\right]^{T}$ is the $4 x 1$ image measurement vector $((l, s)$ for each of two images) with units of pixels;

$X_{0}$ is an a priori estimate of $X$ (updated via iteration of (2));

$M_{0}$ is the a priori predicted measurement based on $X_{0}$;

$B_{X}=\frac{\partial M}{\partial X}$ is the $4 x 3$ partial derivative matrix of the measurements with respect to $X$ and evaluated at $X_{0}$;

$B_{S}=\frac{\partial M}{\partial S}=\left[\begin{array}{cc}B_{S 1} & 0_{2 x n} \\ 0_{2 x n} & B_{S 2}\end{array}\right]$ is the $4 x 2 n$ partial derivative matrix of the measurements with respect to the $n$ sensor adjustable parameters per image and evaluated at $X_{0}$;

$\Sigma \equiv E\left\{\epsilon M \varepsilon M^{T}\right\}$ is the $4 x 4$ mensuration error covariance matrix with units of pixels-squared;

$C_{S} \equiv E\left\{\epsilon S \varepsilon S^{T}\right\}=\left[\begin{array}{ll}C_{S 11} & C_{S 12} \\ C_{S 12}^{T} & C_{S 22}\end{array}\right]$ is the $2 n x 2 n$ error covariance matrix for sensor adjustable parameters for both images, where $C_{S 12}$ is the $n x n$ error cross-covariance between images 1 and 2 (units variable per definition of the adjustable parameters).

The $n x n$ sensor cross-covariance matrix $C_{S 12}$ is a function of temporal correlation as illustrated in the next subsections. For stereo extraction from imagery with similar scales, $C_{S 12}$ affects the solution error covariance $P_{X}$, but only affects the solution $\hat{X}$ if $C_{S 11}$ projected to image space is significantly different than $C_{S 22}$ projected to image space. (The measurements from the two images are not equal weight.) On the other hand, $C_{S 12}$ always affects $\hat{X}$ if the WLS solution is generalized to use more than two images. Correspondingly, reasonable estimates for temporal correlation (hence, all cross-covariance pairs) are also essential for optimal multi-image block adjustments in the absence of a significant number of highly accurate ground control points. See (Dolloff, 2004) for further discussion on the WLS solution and the importance of temporal correlation for multi-image geopositioning and the adjustment of sensor support data.

\subsection{Assumptions Specific to the Simulation}

A simulation was developed to assess stereo solution predicted accuracy. Although relatively simple for convenience, the simulation captures all relevant effects. Figure 3 presents the assumed coordinate systems applicable to both images of the stereo pair. Although it implies general north-south image scans, results are equally applicable to other directions, such as east-west.

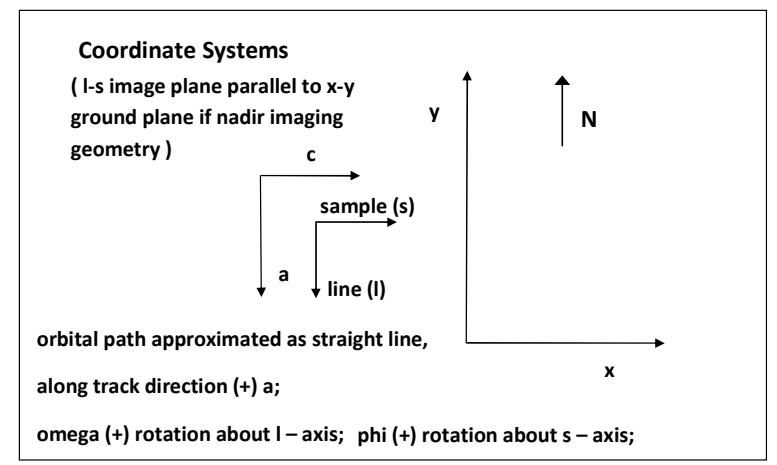

Figure 3. Simulation coordinate systems

Specific to the simulation: the number of sensor adjustable parameters per image $n=5$ corresponding to along-track, crosstrack, radial position components, and Euler angle omega and phi attitude components; imaging geometry is specifiable and expressed as nadir ground sample distance (nadir_gsd), convergence angle (conv), roll angle (roll), and asymmetry angle (asym). Figure 4 presents the stereo imaging angles for the most general scenario. (Note that bie $=\sin ^{-1}(\cos ($ roll $) \cos ($ asym $))$, where bie is the elevation angle of the bisector of the convergence angle.)

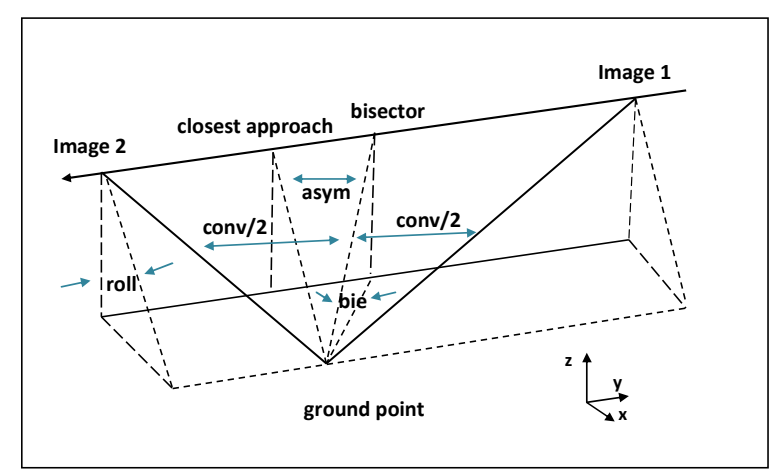

Figure 4. Stereo imaging angles

Although not shown explicitly in Figure 4, imaging geometry includes the orientation of the actual image plane for each image. The (nadir) image plane is rotated about the $\mathrm{x}$-axis (sample-axis) followed by a rotation about the resultant y-axis (line-axis) such that the sensor-to-ground point image ray intersects the principle point, i.e., the image focal axis "points" directly at the ground point.

The computation of the WLS solution's error covariance matrix $P_{X}$ requires the partial derivative matrices $B_{X}$ and $B_{S}$, which are a function of the imaging geometry and the definition of the 
ground coordinates and sensor adjustable parameters, respectively. They are computed numerically for convenience and generality. The computation of $P_{X}$ also requires the mensuration and sensor support data error covariance matrices, detailed as follows:

$\Sigma=\left[\begin{array}{cc}\sigma_{m 1}^{2} I_{2 x 2} & 0_{2 x 2} \\ 0_{2 x 2} & \sigma_{m 2}^{2} I_{2 x 2}\end{array}\right] ;$

$C_{S}=\left[\begin{array}{cccc}C_{a c r 1} & 0_{3 x 2} & \rho_{s p} C_{a c r 1}^{1 / 2} C_{a c r 2}^{T / 2} & 0_{3 \times 2} \\ \cdot & C_{o p 1} & 0_{2 x 3} & \rho_{s a} C_{o p 1}^{1 / 2} C_{o p 2}^{T / 2} \\ \cdot & \cdot & C_{a c r 2} & 0_{3 x 2} \\ \cdot & \cdot & \cdot & C_{o p 2}\end{array}\right] ;$

$C_{a c r i}=\left[\begin{array}{ccc}\sigma_{a i}^{2} & 0 & 0 \\ 0 & \sigma_{c i}^{2} & 0 \\ 0 & 0 & \sigma_{r i}^{2}\end{array}\right]$, and

$C_{o p i}=\left[\begin{array}{cc}\sigma_{o i}^{2} & 0 \\ 0 & \sigma_{p i}^{2}\end{array}\right], i=1,2$

where $\rho_{s p}$ and $\rho_{s a}$ are the scalar temporal correlation values for sensor position and attitude, respectively, $C_{a c r i}$ the $3 \times 3$ a priori error covariance matrix for sensor along-track, cross-track, and radial position adjustable parameters for image $i, C_{o p i}$ the $2 \times 2 a$ priori error covariance matrix for sensor attitude Euler angle rotations omega and phi adjustable parameters for image $i$, and the superscript $1 / 2$ indicating principal matrix square root, and $T / 2$ its matrix transpose. $C_{S}$ is a symmetric matrix, as are all other covariance matrices.

Note that in the above formulation for WLS stereo extraction, sensor adjustable parameters did not include rate terms or a rotation about the image focal axis, aka "kappa". However, for high fidelity operational applications, they may be applicable, depending on the sensor, along with other information such as specific stereo scan directions, e.g., forward/reverse. For other applications that include actual adjustment of the sensor parameters, such as a block adjustment, these terms and other information are typically included.

\subsection{Values Specific to Cases Presented}

Asymmetry was assumed zero for simplicity, and therefore, the bisector elevation angle (bie) is equal to 90 degrees minus roll. For commercial satellite images, asymmetry is typically small (under 10 degrees) and has little effect as discussed in Section 5.

$\sigma_{m 1}=\sigma_{m 2}=1.0$ pixels, sensor height equals 300 nautical miles, and nadir_gsd $=0.5$ meters/pixel;

$C_{S}=\left[\begin{array}{cccc}\sigma_{a c r}^{2} I_{3 \times 3} & 0_{3 x 2} & \rho_{s p} \sigma_{a c r}^{2} I_{3 \times 3} & 0_{3 \times 2} \\ \cdot & \sigma_{o p}^{2} I_{2 \times 2} & 0_{2 \times 3} & \rho_{s a} \sigma_{o p}^{2} I_{2 \times 2} \\ \cdot & . & \sigma_{a c r}^{2} I_{3 \times 3} & 0_{3 \times 2} \\ \cdot \cdot \cdot & . & \sigma_{o p}^{2} I_{2 \times 2}\end{array}\right]$

where $\sigma_{a c r}=0.8 \mathrm{~m}, \sigma_{o p}=2.5$ urad (equivalent to 1.4 meters on the ground at nadir), and $\rho_{s p}=\rho_{s a}$ with specifiable values (unit-less).

\section{EFFECTS OF GEOMETRY ONLY}

This section reviews the well-known effects of imaging geometry (only) by setting sensor uncertainty to zero, nadir_gsd $=1$, and roll angle to zero (or bie $=90$ degrees) for simplicity. The review provides definitions and back ground material in order to better understand the effects of temporal correlation discussed in Section 5.

Recall that $\sigma_{m 1}=\sigma_{m 2}=1.0 \quad$ pixels; therefore, the mensuration error covariance matrix equals the identity matrix $\left(\Sigma=\mathrm{I}_{4 \mathrm{x} 4}\right)$. And because the sensor uncertainty was set to zero $\left(C_{S}=0_{10 \times 10}\right)$, all measurements are uncorrelated and have equal weight $\left(W=I_{4 \times 4}\right)$.

Figure 5 presents WLS solution predicted accuracy as a function of convergence angle and expressed as standard deviations (one-sigma) for ground point coordinates $\mathrm{x}, \mathrm{y}$, and $\mathrm{z}$, computed as the square roots of the diagonal elements of the $3 \times 3$ error covariance matrix $P_{X}$. Because the sensor uncertainty was set to zero, temporal correlation can be considered zero, and results should only be used for relative comparison among the ground coordinates.

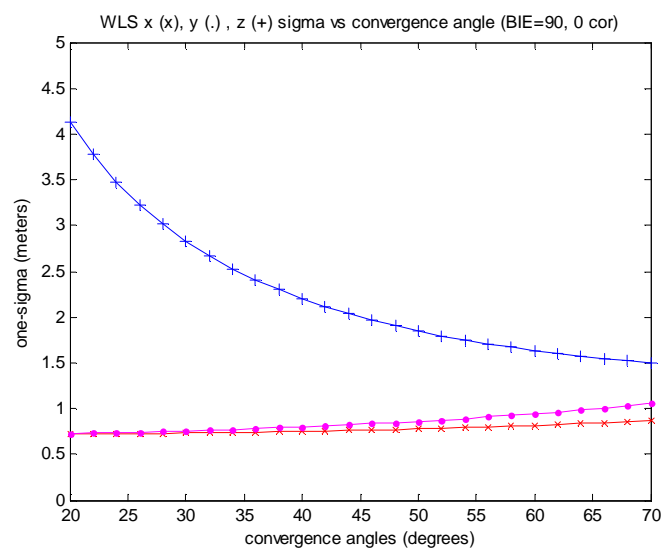

Figure 5. Sigma-x, sigma-y, sigma-z vs. convergence angle

Note that sigma-z $=(b / h)^{-1}$ sqrt(2) gsd_line, corresponding to the geometric effect and the error in the measurement of parallax in the epipolar direction, i.e., $\epsilon Z=(b / h)^{-1}(\varepsilon l 2-$ $\varepsilon l 1)$, where the errors $\varepsilon l 2$ and $\varepsilon l 1$ are uncorrelated, and gsd_line $=$ nadir_gsd $/(\cos (\mathrm{conv} / 2))^{2}$. Figure 6 presents the two conjugate image rays and the corresponding stereo imaging geometry.

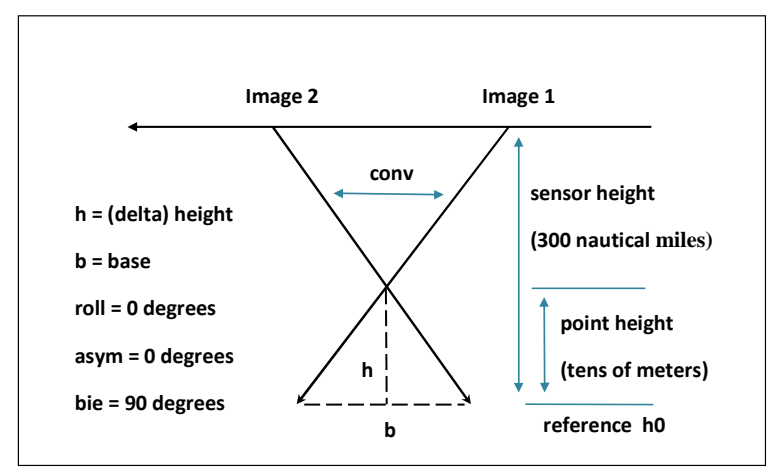

Figure 6. Stereo base (b) and height (h) 
sigma-y = gsd_line $/ \operatorname{sqrt}(2)$, and sigma- $x=$ gsd_sample/ sqrt(2) , where gsd_sample $=$ nadir_gsd/cos (conv/2). The factor 1/sqrt(2) corresponds to two independent (uncorrelated) estimates of horizontal position corresponding to the two image rays.

As demonstrated in Figure 5, sigma-z is very sensitive to convergence angle, as expected. Also, the base-to-height ratio $b / h$ is approximately equal to 0.6 at conv $=35$ degrees, and in accordance with the formula $b / h=2 \tan \left(\frac{\operatorname{conv}}{2}\right)$. Predicted $0.9 \mathrm{p}$ vertical error (LE) and $0.9 \mathrm{p}$ horizontal error (CE) were also computed from the solution's $3 \times 3$ error covariance matrix $P_{X}$. They are plotted as a function of convergence angle in Figure 7, along with their LE/CE ratio. LE equals 1.64 times sigma-z and an approximation for the value of CE is 2.15 times the root-mean-square (rms) of sigma$x$ and sigma-y (however, all CE presented in this paper, including Figure 7, are not approximated and were computed based on the ratio of eigenvalues).

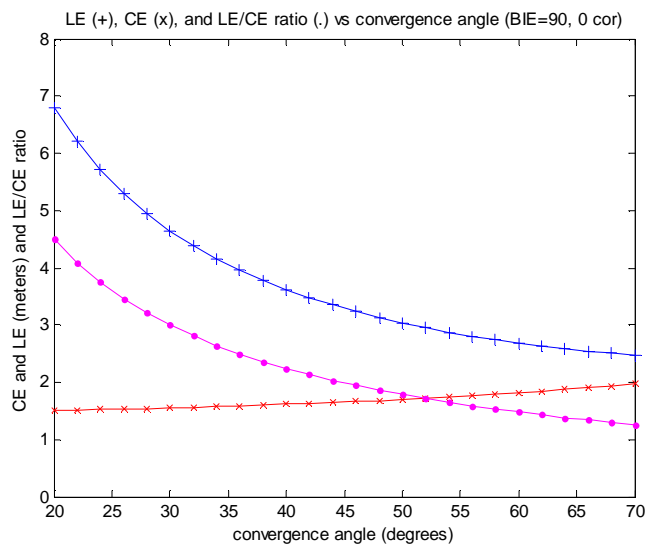

Figure 7. LE, CE, LE/CE vs. convergence angle

\section{EFFECTS OF TEMPORAL CORRELATION}

\subsection{Predicted Accuracy versus Temporal Correlation}

This section demonstrates that temporal correlation of sensor support data errors affects predicted solution accuracy in a manner similar to imaging geometry. Results are based on the WLS stereo extraction algorithm described in Section 3, with temporal correlation specifiable and assumed the same for all sensor adjustable parameters for convenience. The temporal correlation affects the $10 \times 10$ sensor adjustable parameter error covariance matrix $\left(C_{S}\right)$, which in turn affects the $4 \times 4$ measurement weight matrix $(W)$, which in turn affects the solution $3 \times 3$ error covariance matrix $\left(P_{X}\right)$ from which LE and $\mathrm{CE}$ are computed.

More specifically, LE and CE are presented as a function of the amount of temporal correlation (expressed in percent), assuming a fixed imaging geometry typical to commercial satellite imagery. This stereo imaging geometry is the baseline and corresponds to a convergence angle of 37.5 degrees, zero asymmetry, and a roll angle of 15 degrees (bie $=75$ degrees). Together these angles also correspond to an off-nadir angle of 24 degrees (elevation angle of 66 degrees) for each image. The sensor height, nadir gsd, and sensor uncertainties are as described earlier in Section 3.3, and approximate those corresponding to commercial satellite imagery. However, later results are relatively invariant to actual values, as most are based on LE/CE ratios.

Figure 8 presents $\mathrm{LE}$ and $\mathrm{CE}$ as a function of positive temporal correlation, and Figure 9 the corresponding LE/CE ratio. LE decreases significantly with increasing temporal correlation, and $\mathrm{CE}$ increases a lesser amount.

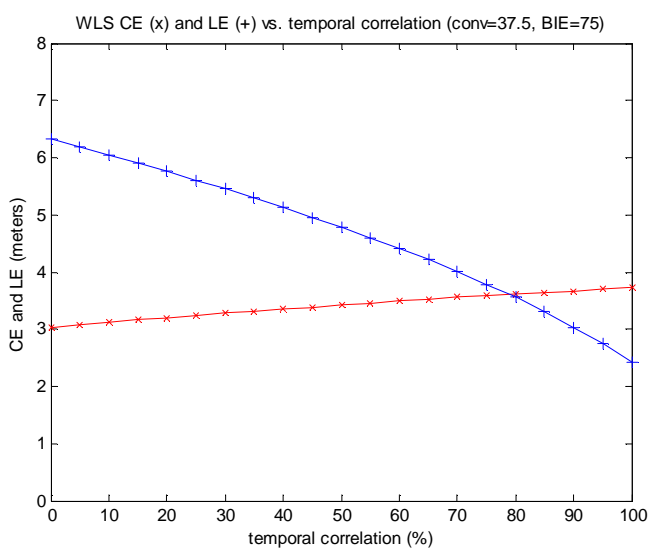

Figure 8. Predicted LE and CE vs. temporal correlation (baseline imaging geometry)

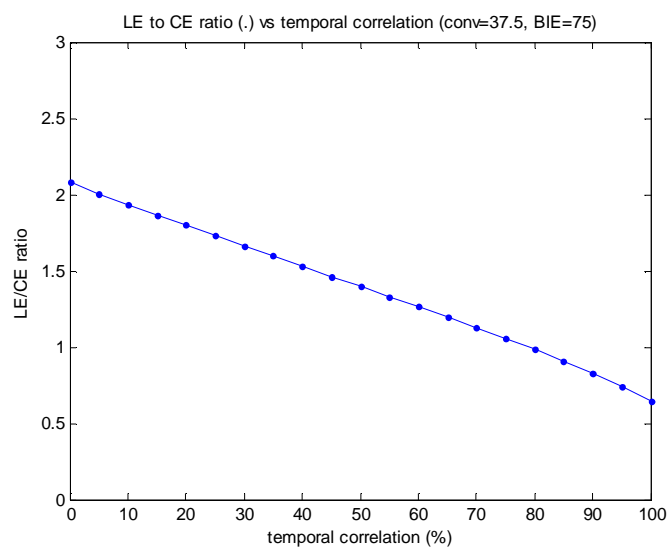

Figure 9. Predicted LE/CE vs. temporal correlation (baseline imaging geometry)

The intuitive reason for improved $\mathrm{z}$ (LE) at higher temporal correlation is the cancellation of common (similar) error, due to sensor support data error, in the two image measurements $((l, s)$ per image). Their scalar difference in the epipolar direction is a measure of corresponding parallax, i.e., directly related to a change in height or elevation. However, there is a related decrease in horizontal accuracy.

\subsection{Derived Value of Temporal Correlation}

Measured values of LE and CE, each computed using numerous stereo pairs and ground truth points, are presented in Table 10 as well as their subsequent ratio. Values correspond to basic stereo pairs, i.e., direct positioning - no use of control. Explicit WLS stereo solutions are not assumed, but rather minimum separation vector solutions; however, these correspond to WLS with equal weight between images, almost always applicable in the real-world and used in the simulation (see Section 3.3) to generate predicted LE and CE. In addition, same-pass images 
were assumed to comprise a stereo pair, which corresponds to nominal imaging operations.

\begin{tabular}{|c|c|c|c|c|c|}
\hline Sensor & LE $(\mathbf{m})$ & CE $(\mathbf{m})$ & LE/CE & \# pairs & reference \\
\hline GE1 & 3.7 & 4.0 & 0.93 & 24 & Bresnahan, 2010a \\
\hline GE1 & 3.2 & 3.4 & 0.95 & 210 & Mulawa, 2010 \\
\hline WV1 & 4.5 & 3.4 & 1.32 & 50 & Dolloff, 2010 \\
\hline WV1 & 5.4 & 4.5 & 1.20 & 25 & Besnahan,2010b \\
\hline
\end{tabular}

Table 10. Measured LE/CE ratios

Comparing the measured results of Table 10 to the predicted results of Figure 9 yields a $60-80 \%$ range for actual temporal correlation, with $70 \%$ a reasonable representative value. It also appears that GeoEye1 has higher temporal correlation than WorldView-1 (approximately 75\% and 65\%, respectively). This may be due to varied sources of error, discussed further in Section 6.1. Further note that, based on Figure 8 and an assumed $70 \%$ actual value for temporal correlation, if WLS stereo extraction mistakenly assumed $0 \%$ temporal correlation between same-pass images, predicted LE would be approximately $60 \%$ too large, and predicted CE approximately $15 \%$ too small.

\subsection{Qualifiers and Potential Future Analyses}

The baseline stereo imaging geometry was assumed for the comparison in Section 5.2. The baseline is compatible with imaging geometry summarized by the image vendors: (DigitalGlobe, 2011) and (GeoEye, 2011). In addition, it is very close to the actual imaging geometry summarized in Table 11 for three of the four measured LE/CE sources of Table 10.

\begin{tabular}{|c|c|c|c|c|c|c|c|}
\hline Sensor & \multicolumn{2}{|c|}{ average (degrees) } & \multicolumn{2}{c|}{ stnd dev (degrees) } & \multirow{2}{*}{ reference } \\
\cline { 2 - 7 } & conv & roll & asym & conv & roll & asym & \\
\hline GE1 & 37 & 11 & 2 & 2 & 7 & 1 & Bresnahan, 2011 \\
WV1 & 39 & 15 & 4 & 3 & 3 & 0 & Dolloff, 2010 \\
\hline WV1 & 35 & 15 & 6 & 4 & 6 & 4 & Bresnahan,2011 \\
\hline
\end{tabular}

Table 11. Stereo imaging geometry for measured LE/CE

The reference (Mulawa, 2010) did not contain imaging geometry and therefore was excluded from Table 11 . The reference (Dolloff, 2010) provided convergence angles and offnadir angles, but did not include asymmetry or roll angles. Therefore, a fixed asymmetry value of 4 degrees was assumed and the off-nadir angles were converted to roll angles consistent with the convergence angles. The reference (Bresnahan, 2011) provided corresponding imaging geometry associated with, but not contained in, both (Bresnahan, 2010a) and (Bresnahan, 2010b). In addition, it included further details: all stereo pairs were same pass, scan directions were the same for the images making up a GeoEye-1 stereo pair, either forward/forward or reverse/reverse, and scan directions were both the same (24\%) and opposite $(76 \%)$ for the images making up a WorldView-1 stereo pair. General scan directions for all GeoEye-1 and WorldView-1 stereo pairs were east-west and north-south, respectively.

The measured LE and CE values in Table 10 are also compatible with the vendors' measured or "typical" values, not their larger specification values.

The predicted accuracy LE/CE ratios of Figure 9 which were used in the Section 5.2 comparison of predicted-to-measured LE/CE ratios were based on the assumption that the same (variable) temporal correlation value was applicable to all sensor support data adjustable parameters, i.e., a "net" or "cumulative" value, for convenience. See Sections 3.2 and Section 3.3 for the specific modeling and Section 6.1 for a related discussion.

The measured results presented in Table 10 correspond to the use of a physical sensor model and its adjustable parameters, not to a corresponding RPC replacement model. However, (Bresnahan, 2010a) and (Bresnahan, 2010b) presents detailed data regarding measured stereo extraction differences between use of the two models that indicate temporal correlation results should change a relatively small amount between models. Specifically, for WorldView1, rms extraction differences between the two models were approximately $0.7 \mathrm{~m}$ for vertical and $0.15 \mathrm{~m}$ for horizontal. These are relatively small and indicate random or uncorrelated polynomial fit errors between same-pass images. (Uncorrelated errors between images allow for an approximate reduction of 1/sqrt(2) in horizontal, and increase of $(b / h)^{-1} \operatorname{sqrt}(2)$ in vertical.) For GeoEye1, rms differences were approximately $0.5 \mathrm{~m}$ for vertical and $1.5 \mathrm{~m}$ for horizontal. Although these indicate a relatively large polynomial fit error, they also indicate highly positively correlated fit errors between same-pass images, e.g. primarily a bias error common to both images.

In general, regarding potential future analyses, more measured data is preferred, including detailed correspondences between measured LE/CE and stereo imaging geometry. Also, measured data corresponding to sensors other than GeoEye-1 and WorldView-1 is desired. Furthermore, the authors plan to investigate the impact that scan direction, the number of imaging operations, and the amount of sensor slewing between the images that comprise a stereo pair have on temporal correlation.

Finally, an alternate approach for the estimation of temporal correlation may also be possible assuming the availability of detailed monoscopic extraction errors relative to ground truth for each image making up each stereo pair for a large sample of stereo pairs. Differences in error per pair are related to temporal correlation.

\subsection{Sensitivities}

The derived value of temporal correlation (Section 5.2) was partially based on the predicted LE/CE ratio as a function of temporal correlation as presented in Figure 9 and corresponding to baseline commercial satellite imaging geometry. In general, for a given value of temporal correlation, the LE/CE ratio increases significantly with decreasing convergence angle and increases somewhat with decreasing roll angle. Thus, to explore the sensitivity of results to perturbations in geometry in "both directions" about the baseline, Figure 12 plots the predicted LE/CE ratio as a function of temporal correlation for three geometry cases: (1) conv $=30$ degrees and roll $=0$ degrees, (2) conv $=37.5$ degrees and roll $=15$ degrees (baseline), and (3) conv $=45$ degrees and roll $=20$ degrees. These three cases also had zero asymmetry and correspond to off-nadir angles of 15 , 24 , and 30 degrees, respectively. Cases one and three correspond to collection extremes for commercial satellite imagery, i.e., their operational limits, whereas case two is the baseline and typical. In addition, Figure 12 contains a case 4, corresponding to the baseline plus 10 degrees asymmetry. As seen in the figure, this level of asymmetry has virtually no effect on LE/CE results; and, although not shown, virtually no 
effect on LE vs. temporal correlation or CE vs. temporal correlation.

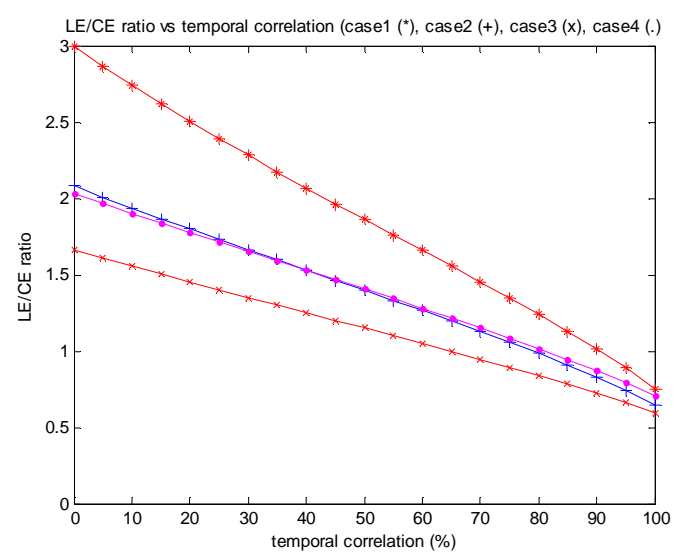

Figure 12. Predicted LE/CE for the baseline, two extreme cases of imaging geometry, the baseline plus asymmetry

If we assume the same measured LE/CE of Table 10, but the corresponding imaging geometry of the relevant case, representative temporal correlation values would become approximately: (1) $85 \%$, (2) $70 \%$, and (3) $55 \%$, for cases $1-3$, respectively. Of course, the assumption of corresponding imaging geometry are incorrect except for case 2, but this analysis serves to bound sensitivity of results to assumptions about corresponding imaging geometry.

\subsection{Temporal Correlation Effect on 3D Error Ellipsoids}

As we have seen in Section 5.1, temporal correlation affects both predicted LE and CE. It follows that it also must affect 3D predicted $0.9 \mathrm{p}$ error ellipsoids, as illustrated in Figure 13 and Figure 14 for the baseline imaging geometry of conv $=37.5$, roll $=15$ degrees, asym $=0$ degrees (bie $=75$ degrees), and for $70 \%$ and $0 \%$ temporal correlation, respectively. These error ellipsoids were generated from the $3 \times 3$ predicted error covariance $P_{X}$ based on the simulation described earlier. Note the incorrect shape of the error ellipsoid if sensor support data errors are incorrectly assumed uncorrelated between same pass images (Figure 14).

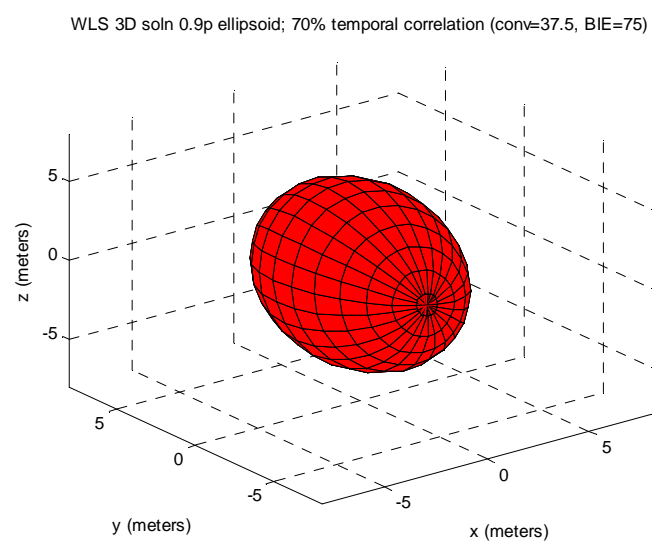

Figure 13. Predicted 0.9p error ellipsoid (70\% temporal correlation)
WLS 3D soln $0.9 p$ ellipsoid; $0 \%$ temporal correlation (conv $=37.5, \mathrm{BIE}=75$ )

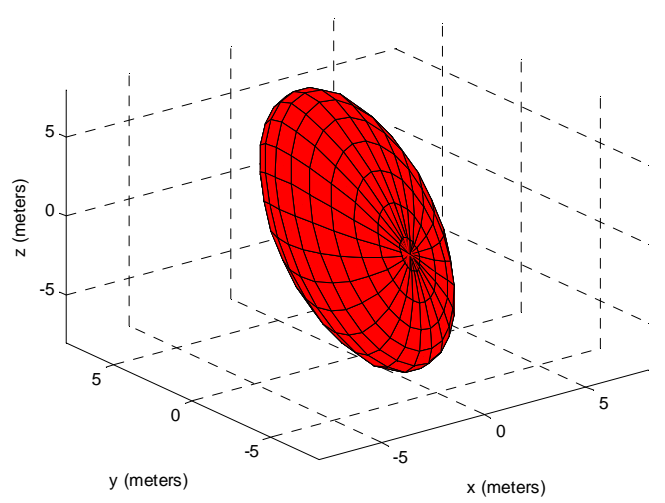

Figure 14. Predicted 0.9p error ellipsoid ( $0 \%$ temporal correlation)

Figure 15 (upper curve) presents the volume of the predicted $0.9 p$ error ellipsoid versus temporal correlation for baseline imaging geometry. For correlation values less than $70 \%$, volume remains relatively constant, indicating a consistent trade-off between vertical and horizontal predicted accuracies. However, higher temporal correlations correspond to a significant decrease in overall predicted 3D location uncertainty (ellipsoid volume) - the higher the positive correlation, the better the overall 3D solution.

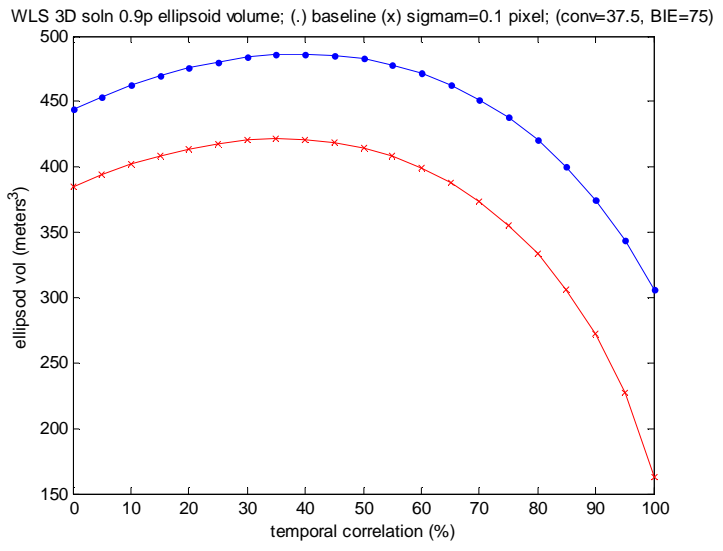

Figure 15. Predicted error ellipsoid volume vs. temporal correlation

This may be somewhat counter-intuitive at first glance; we usually think of independent (uncorrelated) measurements as "good" for a solution. But for stereo extraction, we have four scalar measurements and 3 unknowns, with both a small degree of redundancy "shared" among the three ground coordinates for solution and a somewhat complicated interaction between the three coordinates and the four measurements. For example, recall that the $\mathrm{z}$ coordinate is essentially the scaled difference between two measurements.

This phenomenon becomes even more apparent when reducing the uncertainty of the mensuration error, which we assume uncorrelated, from a one-sigma value of 1 pixel to 0.1 pixel. (Recall that relevant errors are the combination of sensor support data errors and mensuration errors.) Results correspond to the lower curve in Figure 15. Overall, this phenomenon serves to illustrate the important effects of correlation and the need to correctly account for it. 


\section{SOURCES AND REPRESENTATION OF TEMPORAL CORRELATION}

\subsection{Sources of Temporal Correlation}

Sensor position and attitude are typically estimated by Kalman filters or smoothers prior to their insertion into the image metadata stream. Inputs to these estimators include GPS measurements (or derived sensor position estimates) and star tracker measurements of attitude. Because a common set of measurements is used to estimate the metadata stream, metadata errors are correlated by definition - usually highly positively correlated for two points close together in time. In addition, sensor positions based on GPS measurements are inherently positively correlated over reasonably small time intervals due to both orbital dynamics and measurements from a relatively constant set of GPS satellites over the interval.

In general, the metadata errors due to GPS measurements and due to star tracker measurements affect the sensor position and attitude of same-pass images essentially the same way geometrically, i.e., they are "biases" over reasonably short time intervals. For example, if these metadata errors shift the first image (footprint) 2 meters to the northeast, they also shift the second image approximately the same magnitude and direction. Thus, relative to WLS stereo extraction, these metadata errors are positively correlated. This is true regardless the scan directions of the images.

However, there may also be metadata errors associated with knowledge of scan rates or the start time of scans, such as an attitude error due to a time bias (scan rate multiplied by time bias). And reasonably assuming that these metadata errors are positively temporally correlated as well, their effect on WLS stereo extraction accuracy is dependent upon the scan directions for the two images. For two images scanned in the same direction (forward/forward or reverse/reverse), their geometric effect is approximately the same, i.e., corresponds to positive correlation relative to WLS stereo extraction ; for two images scanned in different directions (forward/reverse or reverse/forward) their geometric effect is approximately opposite, i.e., corresponds to negative correlation relative to WLS stereo extraction. The latter tends to reduce the overall positive correlation value of combined metadata errors relative to WLS stereo extraction. The actual effect on the various solution ground coordinates is also a function of the general scan direction, i.e., north-south (approximate epipolar direction) vs. east-west (perpendicular to approximate epipolar direction). The largest effect on the vertical ground component corresponds to a north-south general scan direction.

\subsection{Representation of Sensor Uncertainty and Temporal Correlation}

The metadata for commercial satellite imagery also includes uncertainty (error covariance) of the sensor support data, for both the physical and the RPC sensor models. For the physical sensor model, both image-specific values contained in the metadata, and image-independent default values associated with a sensor model instantiation are available. Currently, values appear overly pessimistic, particularly for the former. In addition, RPC uncertainty parameters are included with RPC metadata. They too, appear overly pessimistic. Of course, the authors expect these values to become more realistic over time. However, neither sensor model contains a description of corresponding temporal correlation (error cross-covariance).
The Sensor Geopositioning Center is working with various image vendors regarding all of the above issues. See (Dolloff, 2012a) for further discussion, particularly concerning the generation and use of RPC uncertainty parameters. The following discusses an approach to represent temporal correlation.

Temporal correlation can be conveniently represented by strictly positive definite correlation functions (spdcf) using only a few parameters. For example, the decaying exponential $e^{-\Delta t / T}$ discussed in Section 2 is one example of an spdcf family, and its time constant $T$ the only parameter needed in order to represent a specific member of the family. There is also a method to approximate the error cross-covariance matrix for sensor position and/or sensor attitude for two images of interest, given an spdcf and the error covariance matrices for the two images. The spdcf is a scalar function of time between images, and the two error covariance matrices need not have the same values or be diagonal. The properties of an spdcf are such that the assembled (full) error covariance matrix is always valid and positive definite. (Section 3.2 presents a simple example of cross-covariance assembly, assuming the values of temporal correlation were obtained by evaluation of two spdcf, one applicable to all sensor position components and one to all attitude components, with the two component groups assumed uncorrelated.)

The reference (Dolloff, 2006) describes various spdcf and the method to compute the corresponding cross-covariance matrix. There can be a different spdcf for each uncorrelated group of sensor adjustable parameters. The image vendor could publish the appropriate spdcf (parameters) for each sensor and possibly each group of sensor adjustable parameters. Their spdef could be generated by the vendor either empirically from data or from specific cross-covariance information that can be generated by augmenting corresponding sensor support data estimators (Kalman filters or smoothers), as described in (Dolloff, 2012b).

An spdcf should change infrequently; hence, its publication via image vendor website or e-mail should suffice. As opposed to an error covariance or cross-covariance matrix, the spdcf is a normalized function (values between -1 to 1 ). Thus, we expect that, while the error covariance may change somewhat between two images on the same-pass, their temporal correlation remains relatively invariant. Of course, its representation and use are an approximation relative to supplying information in the metadata allowing for the exact assembly of a crosscovariance matrix. On the other hand, publishing an spdcf representation of temporal correlation requires no metadata bandwidth.

Figure 16 presents various examples from four different spdcf families (decaying exponential (de), damped cosine (dc), linear decay with positive floor (ldpf), and second order GaussMarkov(gm2)). As seen in Figure 16, spdcf families can have significantly different characteristics, allowing for the selection of one, or the weighted sum of more than one, functional form for a given sensor of interest. 


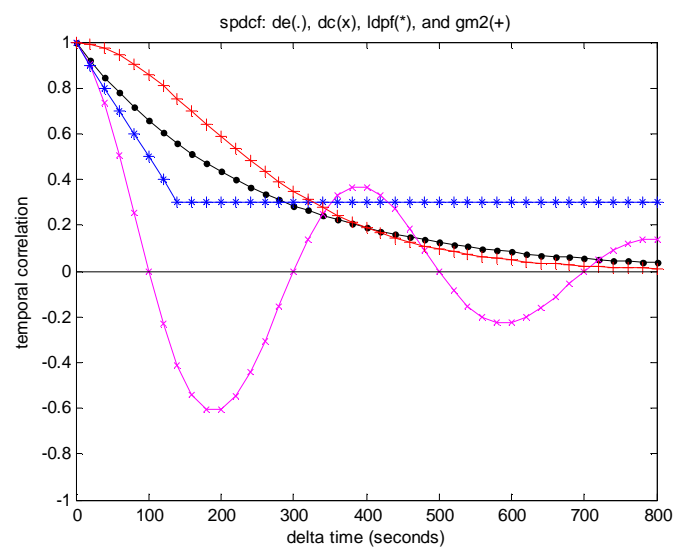

Figure 16. Four examples of spdcf families

\section{CONCLUSIONS}

This paper has addressed the phenomenon that observed vertical errors are much smaller than expected for commercial satellite stereo extraction based on imaging geometry alone. The temporal correlation (similarity) of metadata errors is consistent with this phenomenon.

Through simulation, the significant effects of temporal correlation on predicted stereo extraction accuracy were demonstrated. In addition, based on measured stereo extraction errors, a reasonable case was made for a nominal value of $70 \%$ for the temporal correlation of commercial satellite same-pass imagery as represented by WorldView-1 and GeoEye-1. This value also corresponds to a typical time, about 90 seconds, between images making up the stereo-pair, and in general, will increase somewhat if the time interval is shorter and decrease somewhat if longer. Also, based on the measured data, temporal correlation for GeoEye-1 appears higher than that for WorldView-1 - approximately $75 \%$ vs. $65 \%$.

Many geopositioning applications require near real-time as well as reliable accuracy prediction capabilities. An optimal WLS stereo extraction algorithm was detailed that provides just such a capability. It not only accounts for imaging geometry, it accounts for mensuration error, image metadata (sensor position and attitude) error, and the temporal correlation of metadata errors between same-pass images. Corresponding accuracy predictions do not rely on general or typical imaging geometry, but account for the actual imaging geometry of the stereo pair. Nor do they rely on empirical, generic, non-real time comparisons to ground truth - almost always done independently of the particular stereo pair.

If the representative $70 \%$ temporal correlation is ignored, predicted stereo extraction accuracy will be approximately $60 \%$ too large (pessimistic) for the vertical component and 15\% too small (optimistic) for the horizontal component. In addition, value-added processes such as block adjustments will be missmodeled and non-optimal.

Likely sources for the temporal correlation of sensor support data (image metadata) errors were also discussed, and an spdcfbased method for its practical functional representation and dissemination was suggested.

\section{REFERENCES}

Bresnahan, P., 2010a. Absolute Geolocation Accuracy Evaluation of GeoEye-1 Basic Stereo Triplets. Civil Commercial Imagery Evaluation Workshop. http://calval.cr.usgs.gov/JACIE_files/JACIE10/Presentations/W edPM/Bresnahan_10_005_JACIE2010_CCAP_Geolocation_A ccuracy_GeoEye1_R2-Approved.pdf (12 Aug. 2011).

Bresnahan, P., 2010b. Absolute Accuracy Re-Evaluation of WorldView-1 Basic 1B Stereo Pairs. Civil Commercial Imagery Evaluation Workshop.

http://calval.cr.usgs.gov/JACIE_files/JACIE10/Presentations/W edPM/Bresnahan_10-

015_JACIE2010_CCAP_Geolocation_Accuracy_WV1_24k_R 2-Approved.pdf (12 Aug. 2011).

Bresnahan, P., 2011. Detailed imaging geometry corresponding to GeoEye-1 and WorldView-1. In: private communication.

DigitalGlobe, 2011.

http://www.digitalglobe.com/ (12, Aug. 2011).

Dolloff, J., 2004. McGlone, J., et al, (Eds.), Manual of Photogrammetry, Fifth edition, ASPRS, Bethesda, MA, pp. 913-925.

Dolloff, J., et al, 2006. Strictly Positive Definite Correlation Functions. In: Proceedings of the SPIE, Vol 6235.

Dolloff, J., Settergren, R., 2010. An Assessment of WorldView-1 Positional Accuracy based on 50 Contiguous Pairs of Stereo Imagery. In: PE\&RS Journal, 76(8), pp. 935943.

Dolloff, J., 2012a. RPC Uncertainty Parameters: Generation, Application, and Effects. Accepted for publication, ASPRS 2012 Annual Conference.

Dolloff, J., 2012b. Kalman Filter outputs for inclusion in video-stream metadata: Accounting for temporal correlation of errors for optimal target extraction. Accepted for publication, SPIE 2012 Defense, Security, and Sensing Conference.

Gelb, A., 1974. Applied Optimal Estimation, MIT Press, Cambridge, MA.

GeoEye, 2011.

http://www.geoeye.com/CorpSite/ (12 Aug. 2011).

Mulawa, D., et al, 2010. On Orbit Geolocation Accuracy and Image Quality of the GeoEye-1 High Resolution Imaging Satellite. ASPRS conference, San Diego, CA. http://geoeye.com/CorpSite/assets/docs/technicalpapers/2010/ASPRS_GeoEye-1_Presentation_2010.pdf 